CHAPTER 7

\title{
7 Selective Interaction with Reinforcing Preference
}

9

11 Akira Namatame*

\section{Abstract}

The performance of a collective system crucially depends on the way they interact as well as how they adapt to others. There are two closely related issues concerning a collective of interacting agents, the forward and inverse problems. The forward problem is to investigate what a collective of interacting agents determines as a complex emergent behavior. The inverse problem is to investigate how the private utility functions of agents can be modified so that their self-interested behavior collectively gives rise to a desired outcome. This paper examines the effects of the combined model of the partner choice and preference reinforcing in order to solve the inverse problem. Agents choose their partners and decide on a mode of behavior when interacting with selected partners. Two types of meta-agents are considered: conformists who behave based on the logic of majority, and nonconformists who behave based on the logic of minority. It is shown that a collection of conformists and nonconformists having the identical preference initially evolves into a collection of heterogeneous agents with diverse preferences and that they achieve both an efficient and equitable outcome.

Keywords: cooperation, heterogeneous agents, preference reinforcement, selective interactions

JEL classifications: A12,C79,D83 


\section{1. Introduction}

3 The following question is often addressed: how does a collection of interacting agents generate global macroscopic orders as a whole? An interesting aspect of

5 interacting agents is emergent property. Natural evolution has created a multitude of systems in which the actions of interacting agents give rise to coor-

7 dinated global information processing. Insect colonies, cellular assemblies, the retina, and the immune system have been often cited as examples of having

9 emergent properties. Emergent property is surprising because it can be hard to anticipate the full consequences of even simple forms of interaction. Emergence

11 also refers to the appearance of global information-processing capabilities that are not explicitly represented in the systems' elementary components or in their

13 interconnection. However, there is no presumption that a population of interacting agents in an imperfect world leads to a collectively satisfactory result.

15 How well agents do for it in adapting to their environment is not the same thing as how satisfactory is an environment they collectively create. While all agents

17 understand the outcome is inefficient, acting independently is powerless to manage an efficient collective outcome.

19 In order to lead collective behavior to be a desirable one, we need to consider two different levels: the microscopic level, where the decisions of the individual

21 agents occur and collective behavior can be observed. We also have to specify how agents interact, respond, adapt, or learn from each other. However, to

23 understand the role of the link between these two levels also remains one challenge (Huberman and Glance, 1993; Helbing, 1995).

25 In examining collective effects, we shall draw heavily on the individual decisions. It might be argued that understanding how individuals make decisions is

27 sufficient to understand collective behavior. Many researchers have pointed out that equilibrium theory does not resolve the question of how people behave in a

29 particular interdependent decision situation. It is often argued, "it is hard to see what can advance the discussion short of assembling a collection of people,

31 putting them in the situation of interest, and observing what they do" (Bala and Goyal, 1998, 2000; Banerjee, 1999). People have preferences, pursuing goals, and

33 they behave in a way that we might call "purposive". We metaphorically ascribe motives to behavior because something behaves as if it were oriented toward a

35 goal. But the purposes or objectives often relate directly to those of others. Therefore their behaviors are also constrained by other people who are pursuing 37 their own purposes.

Schelling (1978) characterized contingent behavior - behavior that depends

39 on what others are doing. What makes contingent behavior interesting and difficult is that the entire aggregate outcome is what has to be evaluated, not

41 merely how each person does within the constraints of her own environment. Although individual decision is important to understand, it is not sufficient to

43 describe how a collection of agents arrives at specific decisions. We attempt to probe deeper understanding of the issue by specifying how they interact with 
1 each other. The greatest promise lies in analysis of situations where many agents behave in ways contingent on one another, and these situations are central in

3 theoretical analysis of linking micro to macro levels of collective decision. The overall collective performance depends crucially on the type of interaction as

5 well as on the heterogeneity of agent preferences. An externality occurs if one cares about others' decisions, and their decisions also affect her own decision.

7 An agent's outcome, whichever way he makes his choice, also depends on the number of agents who choose one way or the other. An interesting problem is

9 then under what circumstances will a collection of agents realize some particular stable situations, and whether they satisfy the conditions of efficiency (Iwanaga

11 and Namatame, 2001, 2002).

There are two closely related issues concerning a collective of interacting 13 agents, the forward and inverse problems (Tumer and Wolpert, 2004). An agent behaves based not only on her preference but also on others' actions. It is also 15 important to consider with whom an agent interacts and how each agent decides her action depending on others' actions. Agents are selfish in the sense that they

17 only do what they want to do and what they think according to their own best interests, their motivations. They necessarily have different sets of goals, moti-

19 vations, cognitive states by virtue of their different histories, the different resources they use, or different settings they participate in, and so on.

\section{2. The inverse problem}

25 Agents myopically adapt their behavior based on their idiosyncratic rule to others' behaviors. In the simplest form of our model, agents are born with fixed

27 preferences. They are also assumed to be rational in the sense that they select their strategy in order to maximize their endogenous preferences as shown in

29 Figure 1(a). However individuals' behavior should be understood in a social setting. In order to understand their behavior, we must observe them within

31 social and cultural environments. The inverse problem consists then to investigate how their endogenous preferences can be modified through interactions

33 and strategy choices in the past as shown in Figure 1(b).

35 Figure 1. The forward and inverse problems (a) Preference determines strategy choice (b) Strategy choice reinforces preference

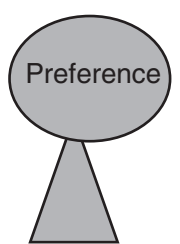

(a)
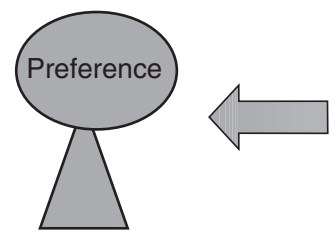

Strategy a)

(b) 
1 It will be nice to motivate the source of learning algorithms by properly redesigning the payment schemes. One possible approach is to consider a me-

3 diator who provides the agents with algorithms to use and suggests payments to be made. This correlation device is not a designer who can enforce behaviors or

5 payments, and it does not possess any private knowledge or aim to optimize private payoffs. Game theory is typically concerned with learning of a strategy

7 (Fudenberg and Levine, 1998). Agents repeatedly play a game, each time observing their rewards, which reflect their prefixed preference. Typically, these

9 studies have the goal of showing that some simple update rule leads the agents to eventually adopt some Nash equilibrium. However, the learning algorithms

11 themselves are not required to satisfy any rationality requirement; it is what they converge to, if adopted by all agents that should be in equilibrium.

13 Shoham and Powers (2003) characterized the so-called 'AI agenda' asking what the best learning strategy is for a given agent for a fixed class of the other

15 agents in the game [19]. It thus retains the design stance of AI, asking how to design an optimal agent for a given environment. There is another agenda that is

17 more prescriptive. It asks how agents should learn in the context of other learners. The recent literature on multi-agent learning also deals extensively with

19 connections between distributed systems and the design of economic mechanisms. One central issue in that literature is the selection of a solution criterion:

21 what can we assume about the agents' rational behavior. Much of the literature deals with the search for mechanisms where the agents will have dominant

23 strategies that lead to desired behavior (maximizing social efficiency). However, it can be shown that such mechanisms rarely exist. One alternative is to consider

25 mechanisms where there exists an ex post equilibrium: a strategy profile of the agents in which it is irrational to deviate from each agent's learning algorithm,

27 assuming the other agents stick to their strategies, and regardless of the state of the system [20].

29

\section{Classification of social interactions}

31

Social interactions pose many coordination problems to individuals. There are

33 many situations where interacting agents can benefit from coordinating their action. Examples where coordination is important include trade alliance, the

35 choice of compatible technologies or conventions such as the choice of a software or language. We can classify interaction with externality into two types.

37 Coordination usually implies that the increased effort by some agents leads the remaining agents to follow them, which rises a multiplier effect or bandwagon

39 effects. These are also characterized as situations where interacting agents can benefit if they take the same action. We call this type as social interaction with

41 positive externalities. In this case, agents behave based on the logic of majority, since agents receive payoffs if they select the same strategy as the majority does.

43 These symmetric social interactions are modeled as coordination games in which an agent receives a payoff if he selects the same strategy as the others. 
1 On the other hand, in the route selection problem for instance, an agents receives a payoff if he selects the opposite strategy as the majority does. For

3 example, in the context of traffic networks, agents have to determine their routes independently. In telecommunication networks, they have to decide what frac-

5 tion of their traffic to send on each link of the network. This type of interaction is distinguished by defining as social interaction negative externalities. In this

7 case, agents behave based on the logic of minority, since agents receive payoff if they select the opposite strategy as the majority does (Beckmann et al., 1956).

9 The El Farol bar problem and its variants minority games provide a clean and simple example of this type of social interaction (Challet and Zhang, 1997).

11 The market entry game is also a stylized representation of a common problem based on the logic of minority: a number of agents have to choose independently

13 whether or not to undertake some activity, such as enter a market, go to a bar, or drive on a road, the utility from which decreasing in the number of the

15 participants (Ochs, 1998; Duffy and Hopkins, 2002). The choice of market entry games for studying coordination is quite natural. When there are too many

17 potential entrants wishing to exploit a new market opportunity, a problem arises regarding how entry may be coordinated. Without coordination, too many firms

19 may decide to enter the market and consequently they sustain losses. Conversely, fully aware of the consequences of excessive entry, firms may be reluctant to 21 enter and exploit the market in the first place. These examples of social interaction typically admit a large number of Nash equilibria. Given this multiplicity

23 of equilibrium outcomes, an obvious question is which type of equilibrium are agents likely to coordinate upon? The coordination failure is attributed to cer-

25 tain features of the payoff functions.

\section{4. Rational decisions of conformists and nonconformists}

29 In most cases, the decision can be thought of as having a positive and negative side - deciding to do a thing or not to do it. We model the problems of in-

31 dividual and collective decisions as follows: each agent has two alternatives, and the costs and benefits of each alternative depend on how many other agents

33 choose the same alternative.

As a specific example, we consider the situation in which each agent in the 35 population of $N$ agents faces the binary decision problem with the following two choices:

$S_{1}$ : votes for,

$S_{2}$ : votes against

One's decision to vote for a particular alternative may depend heavily on how 41 many others decide to do so, partly because of social influence, partly because one does not want to waste her own vote.

43 In this example, the payoff matrix is given in Table 1 . When $\alpha_{i}>0$, agent $i$ personally prefers to vote for, and if $\alpha_{i}<0$, she prefers to vote against. The 
Table 1. The payoff matrix of a conformist

3

Agent $i$

The others

5

$p(t) \quad 1-p(t)$

$S_{1}$

$S_{2}$

9

absolute value of $\alpha_{i}$ represents the strength of her preference. The parameter $11 \beta_{i}(>0)$ represents the level of consistency level with the others' choices.

Agents myopically change their actions based on their own rules obtained as

13 a function of their idiosyncratic utility and of the actions of their neighbors. Assignment of heterogeneous agents also becomes important. We investigate the

15 relation between the collective behavior and the assignment of heterogeneous agents.

17

19 (1) Rational decision of a conformist

We obtain a rational decision rule of a conformist. Let suppose the ratio of agents to choose $S_{1}$ is $p(t)$. We define the threshold of conformist $i$ as:

$$
\theta_{i}=\left(\beta_{i}-\alpha_{i}\right) /\left(\alpha_{i}+\beta_{i}+\alpha_{i}-\beta_{i}\right)=\left(1-\alpha_{i} / \beta_{i}\right) / 2
$$

23 Then the rational decision rule of a conformist is given as:

(i) $\quad p(t) \geq \theta_{i}:$ votes for $\left(S_{1}\right)$

(ii) $\quad p(t)<\theta_{i}$ : votes against $\left(S_{2}\right)$

\section{(2) Rational decision of a nonconformist}

Nonconformist with the payoff matrix of Table 2 prefers the opposite choice

31 of the majority. The threshold of a nonconformist $i$ is defined as:

$$
\theta_{i}=\left(1+\alpha_{i} / \beta_{i}\right) / 2
$$

Then a rational decision rule of a nonconformist is given as:

(i) $p(t)<\theta_{i}$ : votes for $\left(S_{1}\right)$

(ii) $\quad p(t) \geq \theta_{i}$ : votes against $\left(S_{2}\right)$

The strategic decisions of a conformist and a nonconformist can be also

41 modeled with the payoff matrix in Table 3 . If $\beta_{i}>0$, agent $i$ is a conformist, and if $\beta_{i}<0$, she is a nonconformist. From the rational decision rules in (3.2) and 43 (3.4), the agents with heterogeneous payoffs are classified into the following four types. 
Table 2. The payoff matrix of a nonconformist

3

Agent $i$

The others

5

$7 \begin{aligned} & S_{1} \\ & S_{2}\end{aligned}$

$S_{1}$
$S_{2}$

9

Table 3. The payoff matrix of a conformist $\left(\beta_{i}>0\right)$ and nonconformit $\left(\beta_{i}<0\right)$

11

Agent $\mathrm{A}_{i}$

The other agents

13

15

17

$S_{2}$

\begin{tabular}{cc}
\hline$p(t)$ & $1-p(t)$ \\
$S_{1}$ & $S_{2}$ \\
$\alpha_{i}$ & $\alpha_{i}+\beta_{i}$ \\
$\beta_{i}$ & 0 \\
\hline
\end{tabular}

19 (1) Type 1: hardcore $S_{1}$

If agent $i$ is a conformist with the payoff parameters $0 \leq \beta_{i} \leq \alpha_{i}$ or she is a

21 nonconformist with $-\alpha_{i} \leq \beta_{i} \leq 0$, she always chooses $S_{1}$ without regard to the others' decisions. In these cases, the strategy $S_{1}$ becomes a dominant strategy. This type of an agent is a hardcore of the $S_{1}$ chooser.

(2) Type 2: hardcore $S_{2}$

25 If agent $i$ 's payoff parameters satisfy $0<\beta_{i}<-\alpha_{i}$ when she is a conformist, or $\alpha_{i}<\beta_{i}<0$ when she is a nonconformist, she always chooses $S_{2}$ without

27 regard to the others' decisions. In this case, the strategy $S_{2}$ becomes a dominant strategy. This type of an agent is regarded as a hardcore of the $S_{2}$ 29 chooser.

(3) Type 3: conformist

31 If agent $i$ is conformist $\left(\beta_{i}>0\right)$ and her payoff parameters satisfies $\left|\alpha_{i}\right|<\beta_{i}$, her rational decision depends on the proportion of agents who make the same decision. Since he prefers what the majority does, this type of an agent is regarded as a conformist.

(4) Type 4: nonconformist

If agent $i$ is nonconformist $\left(\beta_{i}<0\right)$ and her payoff parameters satisfies $\left|\alpha_{i}\right|<-\beta_{i}$, her rational decision depends on the proportion of agents who make the opposite decision. Since she prefers what the majority does not, this type of an agent is regarded as a nonconformist.

In Figure 2, the $x$-axis represents the parameter value of $\alpha_{i}$, each agent's preference level over two choices (purposive behavior), and the $y$-axis represents the parameter value of $\beta_{i}$, the level of consistency with the others' decisions 
1 Figure 2. Classification of agent types from their idiosyncratic payoffs $\left(\alpha_{i}, \beta_{i}\right)$

3

5

7

9

11

13

15

17

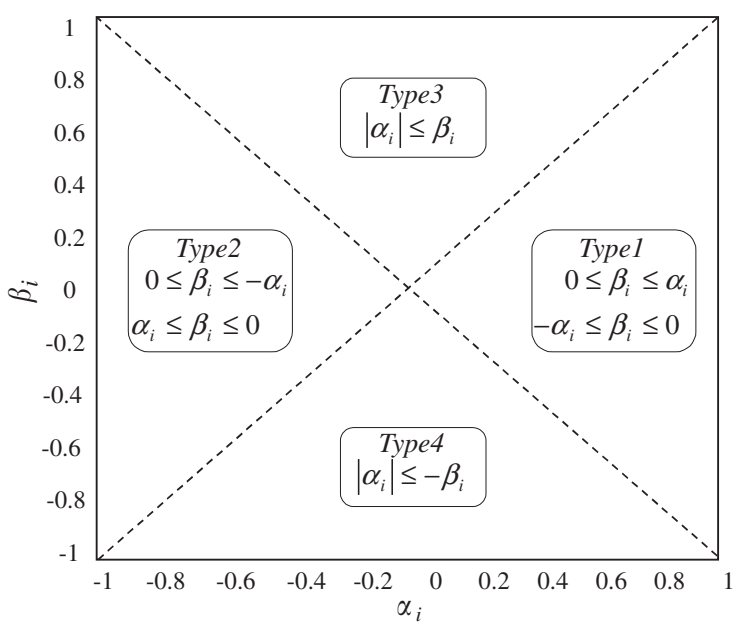

19 (contingent behavior). An infinite number of possible decision rules of heterogeneous agents can be classified into the above four types.

21

\section{Heterogeneity in preferences}

In human societies, an essential element is that individuals differ from each

25 other. The diversity comes into play in many instances of collective behavior. In this section, we depart from the assumption of homogeneity with respect to the

27 payoff parameters. In particular, we consider continuum games with infinite number of heterogeneous agents with respect to their preferences.

29 We assume that a conformist has the payoff matrix in Table 4, and a nonconformist in Table 5. We should remark that the payoff matrices in Tables 1

31 and 2 are strategically equivalent with those in Tables 4 and 5 . The heterogeneity among agents can be described by their payoff parameter $\theta$ ensuring an enor-

33 mous simplification in the present analysis. In our model, agents have idiosyncratic payoff parameter $\theta$. The diversity of a collection of heterogeneous agents is

35 characterized by the distribution function of the payoff parameter $\theta$. We consider a collection of $N$ agents, and denote the number of agents with the same

37 parameter value $\theta$ by $n(\theta)$. We define the density of $\theta$ by $f(\theta)$, which is obtained 39 by dividing $n(\theta)$ by the total number of agent $N$, i.e.

$$
f(\theta)=n(\theta) / N
$$

41 As specific examples, we consider three density functions in Figure 3. In Figure 3(case 1), all agents have the same payoff parameter value $\theta=0.5$, and in

43 Figure 3(case 2), a half of the population have the payoff parameter value $\theta=0$, and the rest of them have $\theta=1$. 
Table 4. The payoff matrix of a conformist

3

\section{Table 5. The payoff matrix of a nonconformist}

$\begin{array}{ll}\text { Choice of agent } i & \text { Choice of other agents }\end{array}$

\begin{tabular}{lcc}
\cline { 2 - 3 } & $S_{1}$ & $S_{2}$ \\
\hline$S_{1}$ & $1-\theta$ & 0 \\
$S_{2}$ & 0 & $\theta$ \\
\hline
\end{tabular}

Choice of other agents

\begin{tabular}{cc}
\hline$S_{1}$ & $S_{2}$ \\
0 & $\theta$ \\
$1-\theta$ & 0 \\
\hline
\end{tabular}

In examining collective effects, we shall draw heavily on the individual adaptive decisions. Within the scope of our model, we treat the case in which agents make deliberate decisions by applying rational procedures, which also guide their reasoning. In order to describe the adaptation process at the individual level, we may have two fundamental models, global interaction and local interaction. It is important to consider with whom an agent interacts and how she decides her action depending on others' actions. Agents may adapt based on the aggregate information representing the current status of the whole system (global information). In this case, each agent chooses an optimal decision based on aggregate information about how all other agents behaved in the past. In many situations, agents are not assumed to be able to correctly guess or anticipate other agents' actions, or they are not able to know how to calculate best replies. With local adaptation, each agent is modeled to adapt to local information (Kirman, 1997; Tennenholtz, 2002).

7 As a specific model, we consider the lattice structure as shown in Figure 4. We arrange a collection of heterogeneous agents in the torus of $50 \times 50(2500)$ agents where the four corners and an edge of an area connect it with an opposite side. The consequence of their actions also gives an effect on agents with whom they are not directly linked. The hypothesis of local adaptation also reflects limited ability of agents to receive, decide, and act based upon information they receive in the course of interaction. 
1 Figure 3. Some density functions of the payoff parameter: $f(\theta)$ Case 1: Distribution with one-peak; Case 2: Distribution with the two-peaks; Case 3: Normal 3 distribution; Case 4: Uniform distribution; and Case 5: Polarized distribution

\section{5}

7

9

11

13

15

17

19

21

23

25

27

31

33

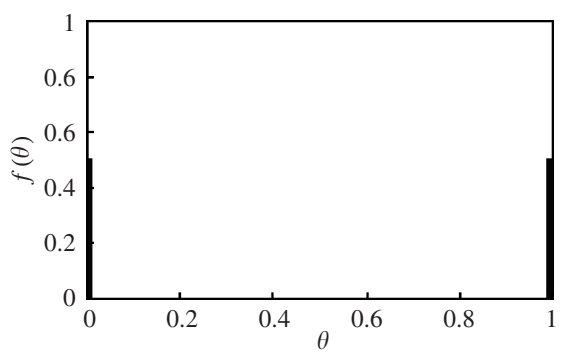

(Case 2)

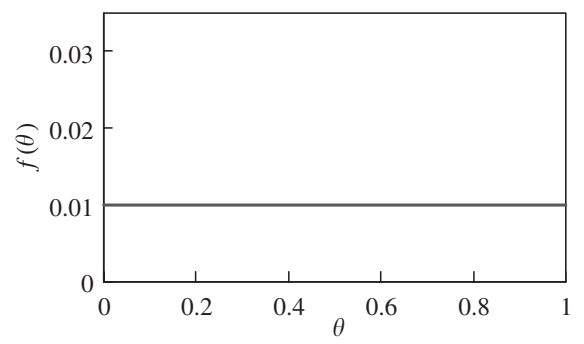

(Case 4)

Global adaptation rule of a conformist

37 Agents adopt actions that optimize their expected payoff given what they expect the others to do. In this model, agents choose the best replies to the

39 empirical frequencies distribution of the previous actions of the others. The main point is that an agent's decision depends on what he knows about the others. We

41 obtain the adaptive rule of each agent as her best response. We denote the proportion of agents having chosen $S_{1}$ at time $t$ by $p(t)$. The optimal adaptive

43 rule of an agent is obtained as the function of the aggregate information on collective $p(t)$ and her idiosyncratic payoff parameter $\theta$ as follows: 
Figure 4. Adaptation to (a) global information and (b) local information

3

5

7

9

11

13

(a)

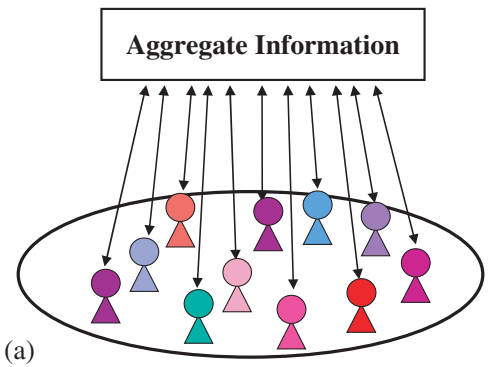

(i) If $p(t) \geq \theta$, choose $S_{1}$

(ii)

If $p(t)<\theta$, choose $S_{2}$.

Local adaptation rule of a conformist

(i)

If $p_{i}(t) \geq \theta$, choose $S_{1}$

(ii)

If $p_{i}(t)<\theta$, choose $S_{2}$.

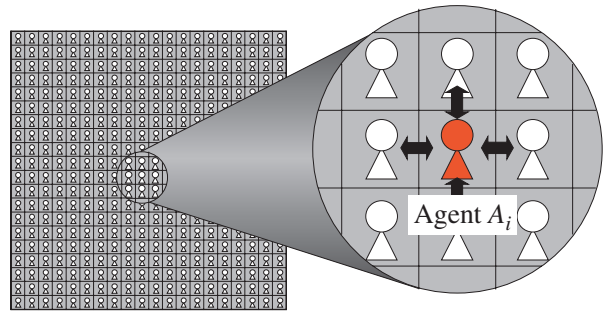

(b)

We denote the proportion of the neighbors of an agent having chosen $S_{1}$ at time $t$ by $p_{i}(t)$. The optimal adaptive rule with local adaptation is obtained as

27 follows:

We evaluate the collective result of interacting heterogeneous agents with two

29 criteria, efficiency and equity. Efficiency is evaluated by the average utility, which also stands for the measure of the desirability at the macro level. Equity is

31 evaluated the utility distribution, which stands for the measure of the desirability at the micro level. The Gini ratio is often used to measure the dispersion of the

33 utility distribution of a society. The pairs of the average payoff $U$ and equity $E$ of all heterogeneous populations of conformists both under global adaptation

35 and local adaptation are shown in Figure 5. The effect of local adaptation compared with global adaptation has been discussed by many researchers (Kir-

37 man, 1997; Tennenholtz, 2002). With these simulation results, both efficiency and equity are low when heterogeneous agents adapt locally. The merit of local

39 adaptation is enhanced for the collective of conformists.

Global adaptation rule of a nonconformist

The global adaptive rules of a nonconformist is obtained as follows:

$$
\text { If } p(t) \leq \theta \text {, choose } S_{1}
$$


1 Figure 5. Efficiency $U$ and equity $E$ for conformists (the initial proportion is $p(0)=0.5)$ (a) Global \& (b) Local adaptation

3

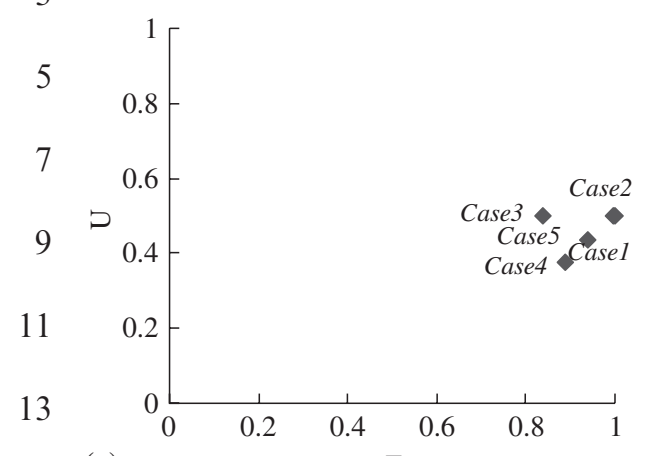

15
(a)
$\mathrm{E}$

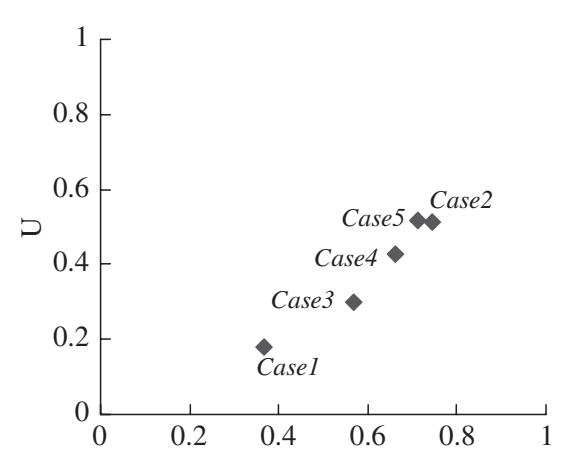

(b)

17 (ii) If $p(t)>\theta$, choose $S_{2}$

Local adaptation rule of a nonconformist

23 The local adaptive rule of a nonconformist is obtained as follows:

(i) If $p_{i}(t) \leq \theta$, choose $S_{1}$

27 (ii)

(ii) If $p_{i}(t)>\theta$, choose $S_{2}$.

29 We evaluate a collective of nonconformists with two criteria, efficiency and equity. The pairs of the average payoff $U$ and equity $E$ of all heterogeneous

31 populations of nonconformists both under global adaptation and local adaptation are shown in Figure 6. As shown in Figure 6, both efficiency and equity

33 are low when conformists adapt locally. On the other hand, the merit of local adaptation is enhanced for the collective of nonconformists.

\section{Selective interaction of heterogeneous agents}

We formalize our idea by modeling a population of heterogeneous agents in

39 which agents are repeatedly matched within a long-time period to play coordination games, see Table 4 . There are many parameters to be considered such

41 as payoff matrix, population structure, population configuration, the number of agents, and so on. Among these parameters, we examine the effects of heter-

43 ogeneity in payoffs $\theta$ and the configuration of locating agents. The interaction methodology also plays an important role in the outcome. We consider two 
1 Figure 6. Efficiency $U$ and equity $E$ for nonconformists (the initial proportion is $p(0)=0.5)$. (a) Global adaptation and (b) Local adaptation

3

5

7

9

11

13

(a)

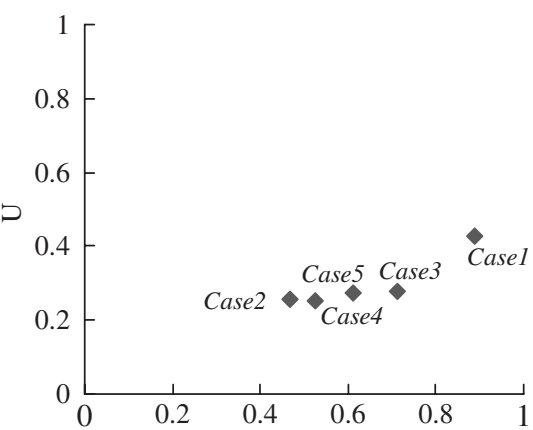

$\mathrm{E}$

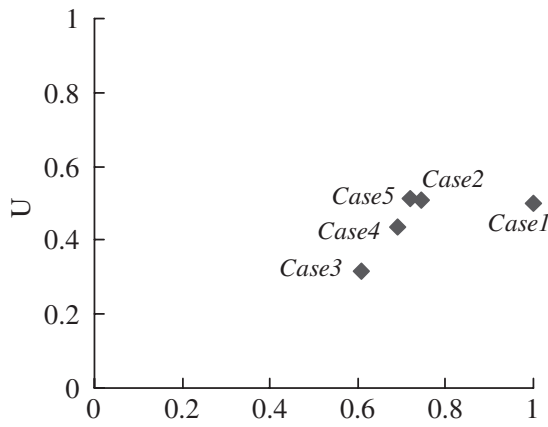

(b)

$\mathrm{E}$

fundamental models, random (or global) interaction and local interaction.

17 Agents myopically evolve their actions based on their own rules obtained as a function of their idiosyncratic utility and of the actions of their neighbors.

19 Assignment of heterogeneous agents also becomes important. We investigate the relation between the collective behavior and the assignment of heterogeneous

21 agents. Our interest is to investigate whether that systems, in which many locally connected processors with no central control, can produce efficient collective

23 performance. We evaluate the collective behavior from the criteria of both efficiency and equity.

25 The crucial concept for describing heterogeneity of agents is their preference characterized by the value of payoff parameter $\theta$. Heterogeneity of preferences

27 makes it possible to have a different type of interaction, selective interaction. This is possible because each agent has a different payoff parameter. We classify

29 heterogeneous agents into the following two types:

Type 1: The payoff parameter value $\theta$ is less than 0.5 (such an agent prefers $S_{1}$ to $S_{2}$ ).

Type 2: The payoff parameter value $\theta$ is greater than 0.5 (such an agent prefers $S_{2}$ to $\left.S_{1}\right)$.

We also classify interaction types into the following three types as shown in Figure 7:

41 (1) Random assignment: each agent has a chance to interact with neighbors of any type.

43 (2) Sorted assignment: each agent interacts with neighbors of the same type.

(3) Mixed assignment: each agent interacts with neighbors of the opposite type. 


\section{Figure 7. Assignment of heterogeneous agents (a) Random, (b) sorting, and (c)} Mixed

3

5

7

9

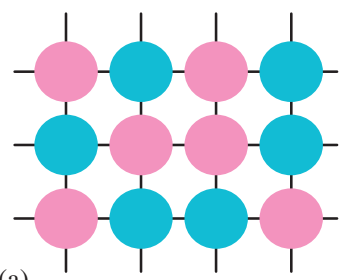

(b)
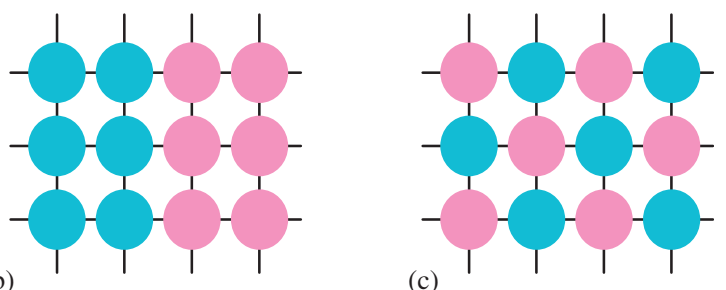

11

13 We describe the selective interaction process of the agents. An agent of type 1 $(\theta \leq 0.5)$ chooses $S_{1}$, since such an agent prefers $S_{1}$ to $S_{2}$. On the other hand, an

15 agent of type $2(\theta>0.5)$ chooses $S_{2}$, since such an agent prefers $S_{2}$ to $S_{2}$. In Figure 8, we represent the selection process of an agent. Each agent interacts

17 with neighbors by choosing her preferred strategy. If she receives the average payoff per one neighbor more than 0.5 , she remains in the same location, oth19 erwise she moves to another location and interacts with different neighbors.

At the beginning, we set two types and heterogeneous agents randomly in the

21 lattice of $50 \times 50$ agents. Conformists and nonconformists of type 1 and type 2 are randomly assigned as shown in Figures 9(a) and 10(a). Each agent interacts

23 with current 8 neighbors. If an agent receives an average payoff per one neighbor less than 0.5 , then he is defined as "unhappy agent". We choose randomly any

25 two unhappy agents on the lattice, and we replace their locations so that all unhappy agents can change their current neighbors. After a few hundreds of

27 rounds, a collection of heterogeneous conformists could self-organize both their locations and their actions so that they can realize homogeneous interaction as 29 shown in Figures 9(b) (conformists) and 10(b) (nonconformists).

We evaluate collectives of both conformists and nonconformists with two 31 criteria, efficiency and equity. The pairs of the average payoff $U$ and equity $E$ under selective interaction are shown in Figure 11. Compared with the results shown in Figure 5, both efficiency and equity are improved when agents interact with the fixed neighbors. Therefore, a collective of heterogeneous agents can

35 achieve the highest efficiency and equity under selective interaction.

\section{7. Selective interaction with reinforcing preferences}

39 Game theory is typically based upon the assumption of a rational choice (Fudenberg and Levine, 1998). The real advantage of the rational-choice as-

41 sumption is that it often allows deduction. The main alternative to the assumption of rational choice is the adaptive approach with reinforcement learning.

43 With reinforcement learning, agents tend to adopt actions that yielded a high payoff, and to avoid actions that yielded a low payoff. Agents will try any of the 
3

5

7

9

11

13

15

17

\section{Figure 8. The process of selective interaction}

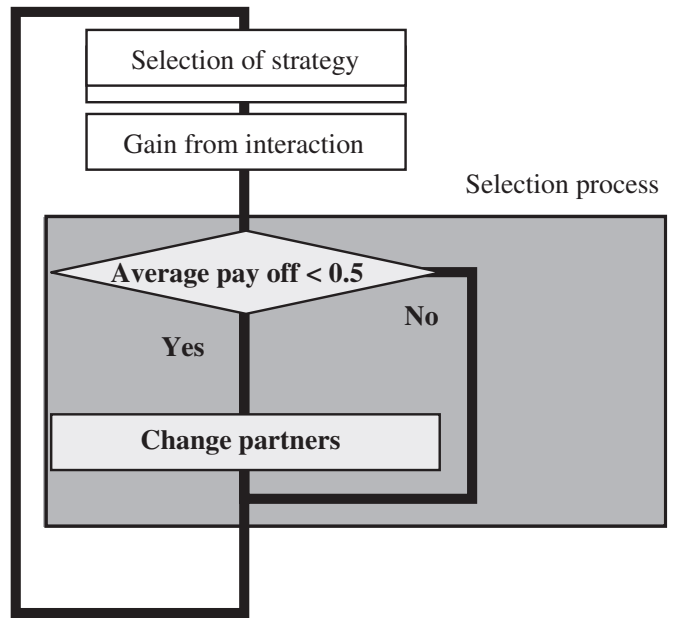

Figure 9. Locations of conformists after selective interactions (a) Random assignment and (b) structured assignment

35 binary choices, and repeat the strategy that led to high payoffs in the past experiences. Propensity of trying a strategy is increased according to the asso-

37 ciated payoff. Payoff describes choice behavior, but it is one's own past payoffs that matter, not the payoffs of the others. The basic premise is that the prob-

39 ability of taking an action in the present increases with the payoff that resulted from taking that action in the past.

41 In this section, we consider a collection of homogeneous agents who initially have the same payoff parameter with $\theta=0.5$ in Table 4 , and the threshold

43 density is shown in Case 1 in Figure 3. Each agent repeats $T(=10)$ interactions with neighbors. If agents receive an average payoff per one neighbor more than 
1 Figure 10. Locations of nonconformist agents after selective interaction (a) Random assignment and (b) structured assignment

3

5

7

9

11

13

15

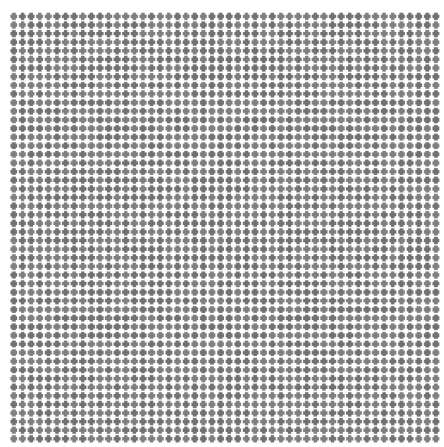

(a)

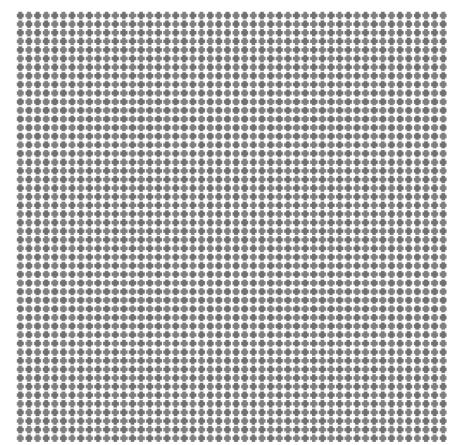

(b)

17 Figure 11. Efficiency $U$ and equity $E$ under structured assignment (a) onformist under sorted assignment and (b) nonconformist under mixed assignment.

19

21

23

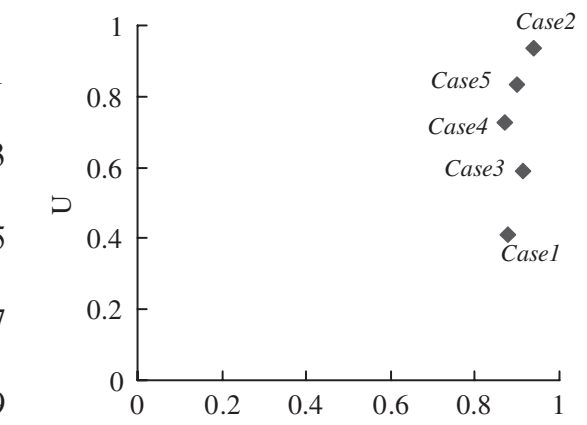

(a)

$\mathrm{E}$

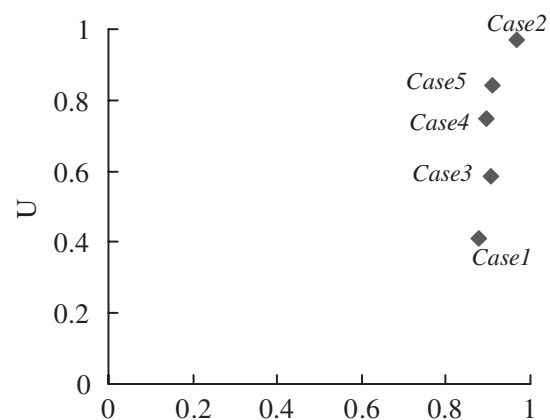

(b)

$\mathrm{E}$

31 Table 6. Reinforcement of learning the payoff-matrix (a) The payoff matrix of a 33 conformist and (b) The payoff matrix of a nonconformist

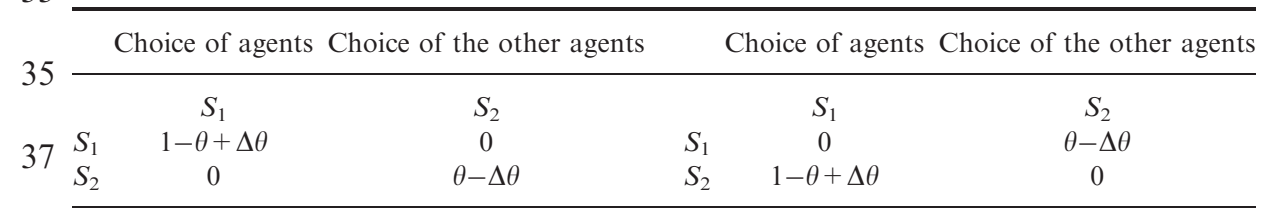

39

0.5 by choosing $S_{1}$, they increase $1-\theta$ by $\Delta \theta=0.01$, hence they decrease $\theta$ by $41 \Delta \theta=0.01$ as shown in Table 6 . Similarly, agents who receive an average payoff more than 0.5 by choosing $S_{2}$, they increase $\theta$ by $\Delta \theta=0.01$. In Figure 12, we 43 represent the processes of collective reinforcement learning. 
1 Figure 12. The reinforcement process of an agent's preference. Count: the number of successful gain

3

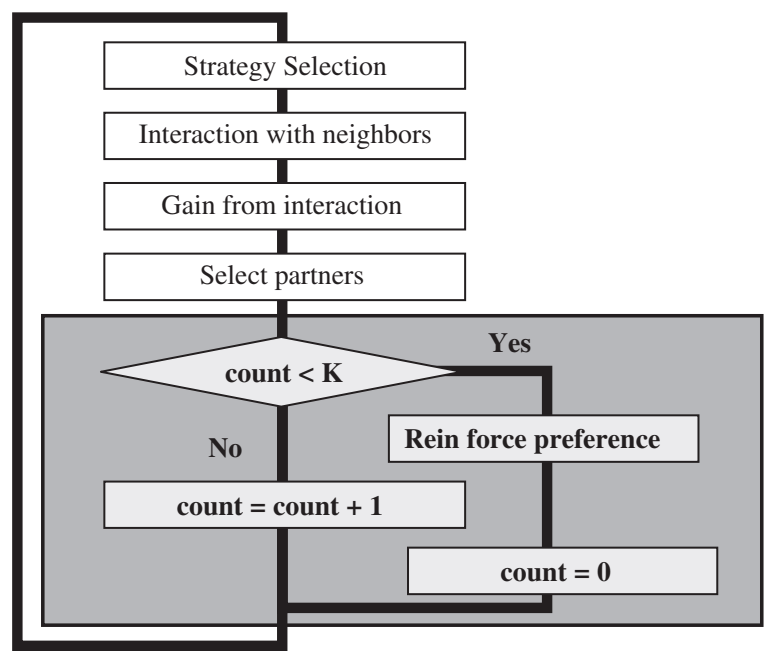

\section{(1) A collective of conformists}

We show the transition of the density function of a collective of conformists in Figure 13. In Figure 13(a), all conformists have the same value $\theta=0.5$ at the beginning, and the initial proportion of agents who choose $S_{1}$ is set to $p(0)=0.25$. A collection of identical conformists gradually self-reinforce their payoff value (preference), and after a repetition of $T=600$ rounds, all agents have the same value of $\theta=1$. In Figure 13(c), the initial proportion $p(0)$ is set to $p(0)=0.5$. Identical conformists with the same payoff parameter $\theta=0.5$ selfreinforce their payoff parameters, and after a repetition of $T=600$ rounds, all agents have the same parameter $\theta=0$. In Figure 13(b), the initial proportion is set to $p(0)=0.5$, and after learning process of $T=600$ periods, a half of agents have the same payoff parameter $\theta=0$, and the rest of agents have $\theta=1$. In all three cases, a collection of identical agents at the beginning collectively reinforce their preferences and evolve into heterogeneous agents, so that the most efficient and equitable collective action can be self-organized. However, the result of collective learning heavily depends on the initial ratio of choosing either strategy.

\section{(2) A collective of nonconformists}

We show the transition of the density function of a collective of nonconformists in Figure 14. Figure 14(a) gives the results when all conformists have the same value $\theta=0.5$ at the beginning, and the initial proportion of agents to choose $S_{1}$ is set to $p(0)=0.5$. A collection of identical conformists gradually self- 
1 Figure 13. Distribution of $\theta$ under reinforcement learning (conformists) (a)

3

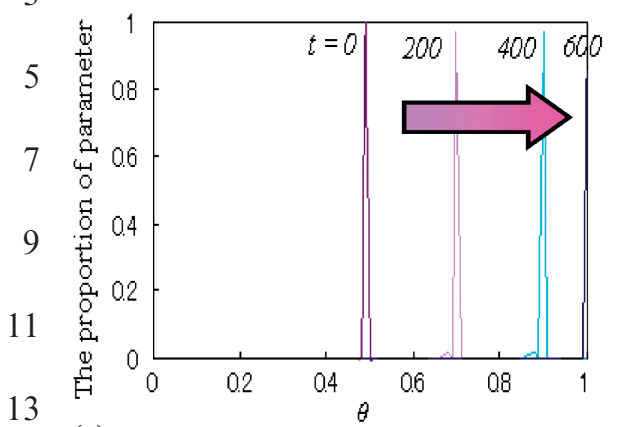

(a)

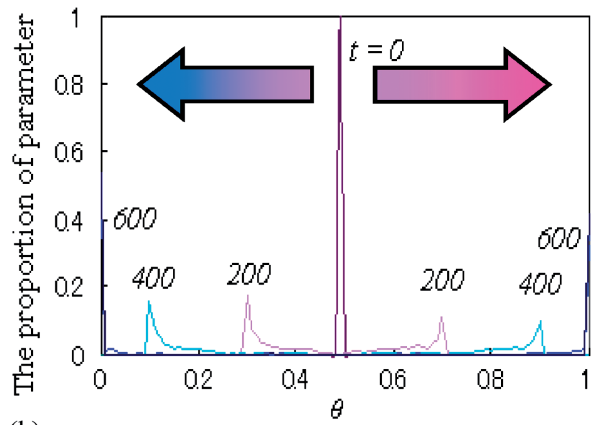

(b)

15

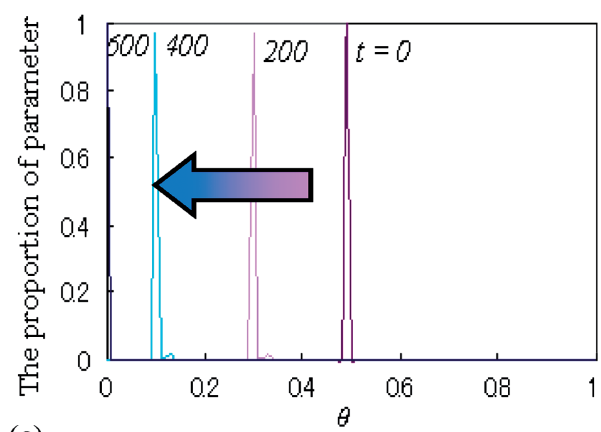

(c)

29 Figure 14. Distribution of $\theta$ under reinforcement learning (nonconformists) (a) $p(0)=0.5(b)(P 0)=0.3$ or $P(0)=0.7$

31

33

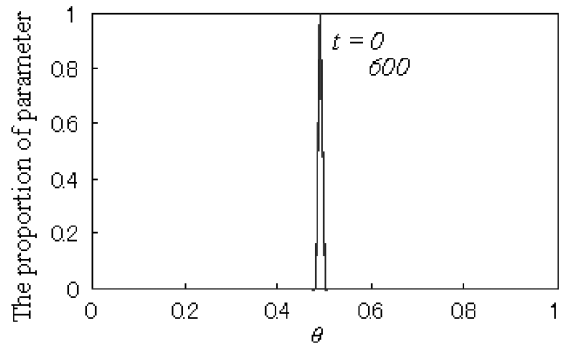

(b)

43 reinforce their payoff value (preference), and after the repetition of $\mathrm{T}=600$ periods, they split into two groups: agents with the payoff parameter $\theta=0$, and 
1 the agents with $\theta=1$. Figure 14(b) shows the results when the initial proportion of agents to choose $S_{1}$ at beginning is set to either $p(0)=0.25$ or $p(0)=0.75$.

3 They remain as homogeneous agents with the same payoff parameter $\theta=0.5$ and they fail to reinforce their preferences. The success of reinforcing noncon-

5 formists' preferences to induce desired collective behavior heavily depends on the initial condition.

\section{(3) A mixed collective of conformists and nonconformists}

We have observed that the success of collective reinforcement learning of conformists and nonconformists heavily depends on the initial condition to choose either strategy. We now consider a mixed case in which conformists who behave with the majority rule and nonconformists who behave with the minority rule coexist in the same population. We assume that the ratios of conformists and nonconformists are the same.

Figure 15 shows the results when the initial proportion of agents to choose $S_{1}$ is set to $p(0)=0.25$. A collection of identical agents who have the same payoff parameter $\theta=0.5$ initially, gradually self-reinforce their preferences, and after the repetition of $T=1000$ periods, $75 \%$ of the agents reinforced the payoff parameter up to $\theta=1$, and the rest of $25 \%$ of agents reinforced to $\theta=0$. The ratio of agents who choose $S_{1}$ is $75 \%$, and all conformists $(50 \%$ of the total population) choose $S_{1}$ and $50 \%$ of nonconformists ( $25 \%$ of the total population) choose $S_{1}$. The ratio of agents who choose $S_{2}$ is $25 \%$, and they are all nonconformists.

Figure 16 shows the results when the initial proportion of agents to choose $S$ is set to $p(0)=0.75$. In this case, a collection of identical agents gradually selfreinforce their payoff parameters, and after the repetition of $T=1000$ periods, $25 \%$ of agents have $\theta=1$, and the rest $75 \%$ of agents have $\theta=0$. The ratio of agents to choose $S_{1}$ is $25 \%$. In this case, all conformists $(50 \%$ of the total

Figure 15. Distribution of $\theta$ under reinforcement learning (conformists and nonconformists with initial ratio $p(0)=0.75$ )

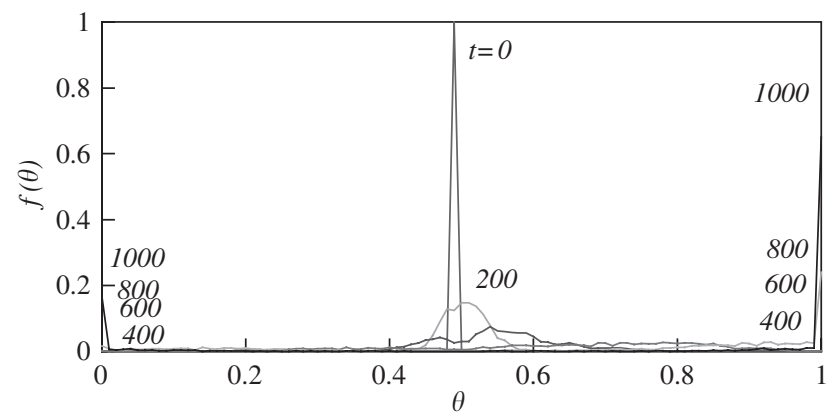


1 Figure 16. Distribution of $\theta$ under reinforcement learning (conformists and nonconformists with initial ratio $p(0)=0.5)$

3

7

9

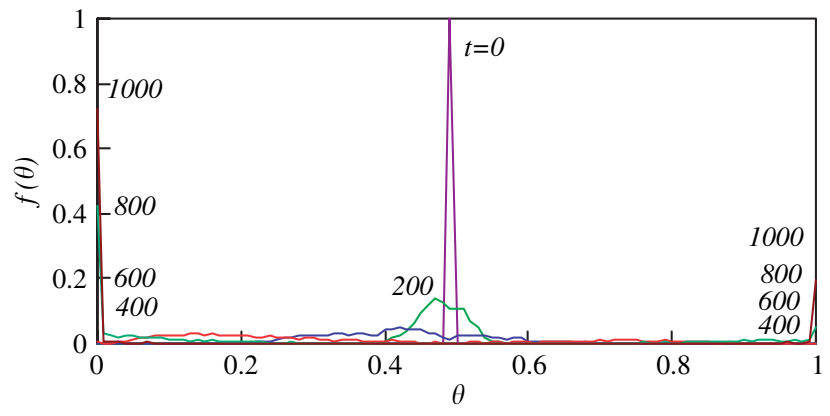

15 population) choose $S_{2}$ and $50 \%$ of nonconformists (25\% of the total population) choose $S_{2}$. The ratio of agents to choose $S_{1}$ is $25 \%$, and they are all nonconformists.

17 Figure 17 shows the results when the initial proportion is set as $p(0)=0.5$. In this case, a half of agents reinforce to have $\theta=1$, and the rest of agents have

$19 \theta=0$. The ratio of agents who choose $S_{1}$ is $50 \%$, and a half of conformists $(25 \%$ of the total population) choose $S_{1}$ and $50 \%$ of nonconformists ( $25 \%$ of the total

21 population) also chose $S_{1}$. The same thing is true for agents who chose $S_{2}$.

In all three cases, a collection of identical agents collectively reinforce their

23 preferences and evolve into heterogeneous agents, so that the most efficient and equitable collective action can be self-organized. The success of collective re-

25 inforcement learning therefore depends on the coexistence of conformists and nonconformists who behave with the opposite rules.

27

29

\section{Conclusion}

There is no presumption that a collective of interacting agents leads to a sat-

31 isfactory performance. Agents normally react to others' decisions, and the resulting volatile collective action is often far from being efficient. In this paper, we

33 investigated how the preferences of agents can be reinforced so that their selfinterested behavior collectively gives rise to a desired outcome. There are many

35 parameters to be considered such as payoff matrix, population structure, population configuration, number of agents, and so on. Among these parameters,

37 we examine the heterogeneity of payoffs and the configuration of locating heterogeneous agents. Agents myopically evolve their actions based on their own

39 rules obtained as a function of their idiosyncratic utility and of the actions of their neighbors. Assignment of heterogeneous agents also becomes to be im-

41 portant. We examined the interaction between partner choice and individual preferences. Agents choose their partners and also decide on a mode of behavior

43 in interactions with these partners. We also investigated how interacting homogeneous agents evolves into heterogeneous agents with diverse preferences by 
1 Figure 17. Distribution of $\theta$ under reinforcement learning (conformists and nonconformists with initial ratio $p(0)=0.25$ )

3

15 reinforcing their preferences so that they can realize the most efficient and equitable collective action. We showed that the most crucial factor that consid-

17 erably improves the performance of the system of interacting agents are the endogenous selection of the partners and reinforcement of preferences at indi-

19 vidual level.

\section{Uncited References}

23 Durlauf and Young, 2001; Hofbauer and Sigmund, 1998; Kollma et al., 1997; Rapoport et al., 1999; Young, 1998.

\section{References}

Bala, V. and S. Goyal (1998), "Learning from neighbors", Review of Economic Studies, Vol. 65, pp. 595-621.

Bala, V. and S. Goyal (2000), "A non-cooperative model of network formation", Econometrica, Vol. 68, pp. 1181-1229.

Banerjee, S. (1999), "Efficiency and stability in economic networks", Mimeo, Boston University.

Beckmann, M., C.B. McGuire and C.B. Winsten (1956), Studies in the Economics of Transportation, Yale University Press.

Challet, D. and C. Zhang (1997), "Emergence of cooperation and organization in an evolutionary game", Physica, Vol. A246.

Duffy, J. and E. Hopkins (2002), "Learning, information and sorting in market entry games", Mimeo, University of Pittsburgh.

Durlauf, S.N. and H.P. Young (2001), Social Dynamics, Brookings Institution Press.

Fudenberg, D. and D. Levine (1998), The Theory of Learning in Games, The MIT Press.

Helbing, D. (1995), Quantitative Sociodynamics, Kluwer Academic. 
1 Hofbauer, J. and K. Sigmund (1998), Evolutionary Games and Population Dynamics, Cambridge University Press.

3 Huberman, B. and N. Glance (1993), "Diversity and collective action", Interdisciplinary Approaches to Nonlinear Systems, Springer.

5 Iwanaga, S. and A. Namatame (2001), "Asymmetric coordination of heterogeneous agents", IEICE Trans. on Information and Systems, Vol. E84-D, pp.

$7 \quad 937-944$.

Iwanaga, S. and A. Namatame (2002), "The complexity of collective decision",

9 Journal of Nonlinear Dynamics and Control, Vol. 6(2), pp. 137-158.

Kirman, A. (1997), "The economy as interactive system", pp. 461-490 in: W.

11 Arthur, editor, The Economy as an Evolving Complex System II, Perseus Books.

13 Kollma, K., J. Miller and S. Page (1997), "Computational Political”, pp. 531 in: W. Arthur, editor, The Economy as an Evolving Complex System II, Perseus

15 Books.

Ochs, J. (1998). "Coordination in market entry games," pp. 143-172 in: D.

17 Budescu, I. Erev and R. Zwick, editors, Games and Human Behavior.

Rapoport, A., D. Seal and S. Winter (1999), "An experimental study of coor-

19 dination and learning in iterated two-market entry games", Economic Theory, Vol. 16, pp. 661-687.

21 Schelling, T. (1978), Micromotives and Macrobehavior, Norton.

Shoham, Y. and R. Powers (2003), "Multi-agent reinforcement learning: a crit23 ical survey", Memo.

Tennenholtz, (2002). "Efficient learning equilibrium", in: Proceedings of NIPS.

25 Tumer, K. and D. Wolpert (2004), Springer.

Young, H.P. (1998), Individual Strategy and Social Structures, Princeton Uni27 versity Press. 


\section{AUTHOR QUERY FORM}

\begin{tabular}{|l|l|l|}
\hline & Please e-mail or fax your responses and any corrections to: \\
\hline ELSEVIER & Chapter : 80008 & E-mail: \\
\hline
\end{tabular}

Dear Author,

During the preparation of your manuscript for typesetting, some questions may have arisen. These are listed below. Please check your typeset proof carefully and mark any corrections in the margin of the proof or compile them as a separate list*.

\section{Disk use}

Sometimes we are unable to process the electronic file of your article and/or artwork. If this is the case, we have proceeded by:
$€$ Scanning (parts of) your article
$€$ Rekeying (parts of) your article
$€$ Scanning the artwork

b Uncited references: This section comprises references that occur in the reference list but not in the body of the text. Please position each reference in the text or delete it. Any reference not dealt with will be retained in this section.

\section{Queries and / or remarks}

\begin{tabular}{|c|l|c|}
\hline Location in Article & \multicolumn{1}{|c|}{ Query / remark } & \multicolumn{1}{|c|}{ Response } \\
\hline AQ1 & $\begin{array}{l}\text { Please provide the place of publication in Becmann et al. (1956); Durlauf and } \\
\text { Young (2001); Fundenberg and Levine (1998); Helbing (1995); Holfbauer and } \\
\text { Sigmund (1998); Huberman and Glance (1993); Kirman (1997); Tumer and } \\
\text { Wolpert (2004); and Young (1998). }\end{array}$ & \\
\hline AQ2 & Please provide page range in Challet and Zhang (1997). & \\
\hline AQ3 & $\begin{array}{l}\text { Please provide the names of all editors in Kirman (1997) and Kollma et al. } \\
\text { (1997). }\end{array}$ & \\
\hline AQ4 & $\begin{array}{l}\text { Please check the number [19] at the end of the sentence in "Shoham and } \\
\text { Powers (2003) characterized .... }\end{array}$ & \\
\hline AQ5 & $\begin{array}{l}\text { Please check the number [20] at the end of the sentence in "One alternative is } \\
\text { to .... }\end{array}$ & \\
\hline & & \\
\hline
\end{tabular}

Thank you for your assistance 